\title{
Activation of Tax protein by c-Jun-N-terminal kinase is not dependent on the presence or absence of the early growth response-1 gene product
}

\author{
EDUARDO PARRA ${ }^{1}$, LUÍS GUTIERRÉZ ${ }^{2}$ and JORGE FERREIRA ${ }^{3}$ \\ ${ }^{1}$ Laboratory of Experimental Biomedicine, University of Tarapaca, Campus Esmeralda, Iquique; \\ ${ }^{2}$ Faculty of Sciences, University Arturo Prat, Iquique; ${ }^{3}$ Programme of Molecular and Clinical Pharmacology, \\ ICBM, Medical Faculty, University of Chile, Santiago, Chile
}

Received July 5, 2015; Accepted August 18, 2015

DOI: 10.3892/or.2015.4424

\begin{abstract}
The Tax protein of human T cell leukemia virus type 1 plays a major role in the pathogenesis of adult $\mathrm{T}$ cell leukemia (ATL), an aggressive neoplasia of $\mathrm{CD}^{+} \mathrm{T}$ cells. In the present study, we investigated whether the EGR-1 pathway is involved in the regulation of Tax-induced JNK expression in human Jurkat T cells transfected to express the Tax protein in the presence or absence of PMA or ionomycin. Overexpression of EGR-1 in Jurkat cells transfected to express Tax, promoted the activation of several genes, with the most potent being those that contained AP-1 (Jun/c-Fos), whereas knockdown of endogenous EGR-1 by small interfering RNA (siRNA) somewhat reduced Tax-mediated JNK-1 transcription. Additionally, luciferase-based AP-1 and NF- $\kappa \mathrm{B}$ reporter gene assays demonstrated that inhibition of EGR-1 expression by an siRNA did not affect the transcriptional activity of a consensus sequence of either AP-1 or NF- $\kappa$ B. On the other hand, the apoptosis assay, using all-trans retinoic acid (ATRA) as an inducer of apoptosis, confirmed that siRNA against EGR-1 failed to suppress ATRA-induced apoptosis in Jurkat and Jurkat-Tax cells, as noted by the low levels of both DEVDase activity and DNA fragmentation, indicating that the induction of apoptosis by ATRA was Egr-1-independent. Finally, our data showed that activation of Tax by JNK-1 was not dependent on the EGR-1 cascade of events, suggesting that EGR-1 is important but not a determinant for the activity for Tax-induced proliferation of Jurkat cells.
\end{abstract}

\section{Introduction}

Human T-cell leukemia virus type 1 (HTLV-1) is the etiologic agent of adult $\mathrm{T}$ cell leukemia (ATL), an aggressive neoplasia

Correspondence to: Dr Eduardo Parra, Laboratory of Experimental Biomedicine, University of Tarapaca, Campus Esmeralda, Avenida Luis Emilio Recabarren 2477, Iquique, Chile

E-mail: eparra@uta.cl

Key words: ATRA, Tax, c-Jun-N-terminal kinase, Jurkat cells, early growth response-1 of $\mathrm{CD}^{+} \mathrm{T}$ cells $(1,2)$. The HTLV-1 transcriptional transactivator protein Tax transactivates the expression of many cellular genes in addition to the viral LTR (3-5). The cellular genes activated by Tax include several involved in cell growth, which suggests that it is the expression of this protein that deregulates T cell growth during HTLV-1 infection and, indeed, the constitutive expression of Tax is correlated with immortalization in $\mathrm{T}$ cells and the transformation of other cell types (6-9). Tax corresponds to a $40-\mathrm{kDa}$ transforming protein $(10,11)$ from the pathogenic retrovirus HTLV-1 that induces the expression of various family members of the transcription factor AP-1, such as c-Jun, JunD, c-Fos, and Fra-1, at the level of RNA expression in T cells $(12,13)$.

The Jun-N-terminal kinase (JNK) is the only member of MAP kinases to phosphorylate c-Jun, the main component of AP-1 complexes, and also has ATF-2 and Elk-1 as substrates $(14,15)$. In mammalian cells, three MAPK families have been clearly characterized: namely the classical MAPK (also known as ERK), C-Jun N-terminal kinase/stressactivated protein kinase (JNK/SAPK) and p38 kinase. The JNK group of mitogen-activated protein kinases (MAPKs) is activated in response to the treatment of cells with inflammatory cytokines and by exposure to environmental stress. JNK activation is mediated by a protein kinase cascade composed of a MAPK kinase and a MAPK kinase kinase (14-16). JNK and p38 kinases were initially proposed to mediate apoptosis in neuronal cells (17), and phosphorylation of c-Jun is necessary for neuronal cell death (18). The use of kinase inhibitors and overexpression of dominant-negative mutant forms of MAPKs have demonstrated a role of JNK and/or p38 kinase in apoptosis induced in non-neuronal cells by various stimuli, including estrogen, cisplatin, UV-B radiation, and singlet oxygen $(19,20)$. Recently, a link between EGR-1 and JNK has been demonstrated (21), and JNK-1 was found to be able to regulate the expression of EGR-1 (22). Although EGR-1 was cloned and characterized as an 'immediate-early response' gene, several studies have shown that EGR-1 plays a functional role in the regulation of growth and suppression of the transformation of several types of human cancer cells $(23,24)$. Moreover, the majority of primary human glioblastoma and astrocytomas show decreased or deleted 
expression of EGR-1, indicating a potential growth suppressive role in human glioblastomas $(25,26)$ and small cell lung carcinoma (27). EGR-1 rapidly activates signal transduction pathways involving the MAPKs that activate the Egr-1 promoter. Inducible Egr-1 gene expression is mediated in part by the extracellular signal-regulated kinase (ERK), c-Jun NH2-terminal kinase (JNK) and p38 pathways.

Our results revealed that EGR-1 is somewhat detectable in quiescent human Jurkat cell lines. This expression is strongly enhanced by PMA treatment. Blocking of EGR-1 expression by a specific siRNA did not affect the expression of JNK induced by Tax in Jurkat cells transfected to express the Tax protein. Finally, our data showed that EGR-1 is constitutively activated in Jurkat leukemia T cells expressing the Tax protein, suggesting that EGR-1 is important but not a determinant for the activity for Tax-induced proliferation of Jurkat cells.

\section{Materials and methods}

Reagents. The protease inhibitors phenylmethylsulfonyl fluoride (PMSF), leupeptin, pepstatin, aprotinin, EDTA and bestatin were purchased from Roche (USA); T4 polynucleotide kinase and poly $(\mathrm{dI}-\mathrm{dC}) 2$ were obtained from Amersham Pharmacia Biotech (Piscataway, NJ, USA). Tris-borate-EDTA buffer and acrylamide-bisacrylamide (29:1) were obtained from Bio-Rad (Richmond, CA, USA). Luciferase assay reagent, lysis buffer and the pGL-3 luciferase vector were obtained from Promega (Madison, WI, USA). All-trans-retinoic acid (ATRA), dimethyl sulphoxide (DMSO), Triton X-100, 1,4-dithiotreitol (DTT), paraformaldehyde, protease inhibitor cocktail, phosphatase inhibitor cocktail, Tween-20, Temed, $30 \%$ acrylamide, TPA and ionomycin were purchased from Sigma (USA). Anti JNK-1, -JNKp, -EGR-1, - $\beta$-actin, -HTLV-1 Tax (sc-34096) antibodies were purchased from Santa Cruz Biotechnology (Santa Cruz, CA, USA). Cell Death Detection ELISA was purchased from Roche (Indianapolis IN, USA).

Cell culture. Jurkat T cells and Jurkat T cells transfected to express Tax protein, were grown in RPMI-1640 medium containing $10 \%$ heat-inactivated FBS, $200 \mathrm{mM}$ glutamine, non-essential amino acids, penicillin, and streptomycin sulfate. Before treatment, the cells were grown overnight (16-20 h) in medium containing $0.5 \%$ heat-inactivated FBS and subsequently stimulated in the presence of the same medium with low concentrations of serum. ATRA was diluted in culture medium containing $0.5 \%$ FBS, keeping the DMSO concentration below $0.5 \%$. Appropriate controls containing the same amount of solvent were included in each experiment. Intermittent passage in G418-containing medium was performed to ensure retention of the plasmid (28).

Plasmid construction and preparation of nuclear extracts. The plasmid expressing the wild-type of the Tax protein and the Tax-inducible cell line, were a gift from Dr Warner Greene (Gladstone Institute of Virology and Immunology, University of California, San Francisco, San Francisco, CA, USA). The human IL-2 promoter-enhancer fragment $(-500$ to +60$)$ was subcloned from plasmid SV-IL-2-CAT into the luciferase vector pGL-2 (Promega) (28). The AP-1-luciferase reporter plasmid driven by the rat prolactin minimal promoter $(-36$ to
+37) under the control of four copies of the human AP-1 site (28) was kindly provided by M. Rincón and R.A. Flavell (Section of Immunobiology, Howard Hughes Medical Institute, Yale University School of Medicine, New Haven, CT, USA). Plasmids containing multimers of the recognition sites for $\mathrm{NF}-\kappa \mathrm{B}$ and AP-1 were constructed and linked to the pLuc-prolactin minimal promoter plasmid (28). The orientation for each element was confirmed by restriction enzyme cleavage. The tandem sequences used to construct the different multimer plasmids were as follows: i) four copies of the AP-1-responsive element (the 12-O-tetradecanoylphorbol-13acetate [TPA]-responsive element of the human collagenase promoter), (5'-TCGATTGAGTCAGGGTAA-3'), and ii) two copies of the NF- $\kappa$ B-binding site of the human Ig $\kappa$ light chain enhancer (5'-GGGACTTTCC-3') (28).

siRNA preparation and transfection of small interfering RNA. The siRNAs for Egr-1 and JNK-1 were obtained as ready-annealed, purified duplex probes, and the scrambled control siRNAs were purchased from Shanghai Genechem Co.: siRNA for Egr-1 (sense, 5'-CAGCAGCAGCAGCAGCAG CTT-3' and antisense, 5'-AAGCTGCTGCGCTGCTGCTG-3'); siRNA oligonucleotides for JNK-1 (sense, 5'-AAGCCCAGTA ATATAGTAGTA-3' and antisense, 5'-TACTACTATATTACT GGGCTT-3'). The cells were cultured in medium without antibiotics and $24 \mathrm{~h}$ before transfection resulting in confluency of the cell monolayer by 50-70\%. Specific EGR-1 and JNK-1 siRNAs or non-silencing siRNA $(70 \mathrm{nmol})$ were mixed with Lipofectamine $^{\mathrm{TM}} 2000$ (Invitrogen) according to the manufacturer's recommendation and added to the cells. After $6 \mathrm{~h}$ at $37^{\circ} \mathrm{C}$, the medium was changed, and the cells were cultivated in RPMI-1640 medium supplemented with $10 \%$ heat-inactivated FBS.

Cell proliferation assay. Cell survival was measured using a 3-(4,5-dimethylthiazol-2-yl)-2,5-diphenyltetrazolium bromide (MTT) cell proliferation assay. Cells (10,000/well) were seeded in 96-well plates in $100 \mu \mathrm{l}$ of medium containing the appropriate amount of serum. Jurkat and Jurkat-Tax cells were transfected or not with siRNA-control or siRNA-Egr-1 and were then treated with ATRA $8 \mu \mathrm{M}$ or solvent (DMSO) for 1 , 2,4 or 6 days, when $10 \mu \mathrm{l} /$ well of $5 \mathrm{mg} / \mathrm{ml}$ MTT was added. After $4 \mathrm{~h}$ of incubation at $37^{\circ} \mathrm{C}$, the tetrazolium crystals were solubilized overnight at $37^{\circ} \mathrm{C}$ with $10 \%$ SDS and $10 \mathrm{mM} \mathrm{HCl}$, and the optical density (OD) at $595 \mathrm{~nm}$ was measured.

Transient transfection and luciferase assays. Transfection of cells was conducted by electroporation, using an Electro Cell Manipulator 600 (BTX, San Diego, CA, USA) using

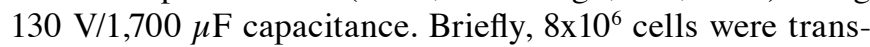
fected with $10 \mu \mathrm{g}$ of luciferase reporter plasmid and $5 \mu \mathrm{g}$ of each expression plasmid, and the mixture was incubated for $24 \mathrm{~h}$. Transfected cells were cultured in complete medium for $24 \mathrm{~h}$ and stimulated with ATRA for another $8 \mathrm{~h}$. Cells were harvested $32 \mathrm{~h}$ post-transfection, washed twice in PBS, and treated with lysis buffer (Luciferase assay; Promega) for 5 to $10 \mathrm{~min}$ on ice. Lysates were spun down for $1 \mathrm{~min}$, and the total supernatants were analyzed using luciferase reagent (Promega) and measured as a duplicate in a luminometer (MicroLumat LB 96 P; Berthold) for 5 sec. Background measurement was 
subtracted from each duplicate, and experimental values are expressed either as recorded light units of luciferase activity or as relative activity compared with extracts from unstimulated cells (29). Nuclear extracts were prepared as previously described $(29,30)$.

Western blot analysis. The Jurkat cell line $\left(5 \times 10^{7}\right)$ was seeded onto 6-well plates. Forty-eight hours after transfection, the cells were collected and washed twice by cold PBS, and each well was treated with $50 \mathrm{ml}$ lysis buffer ( $2 \mathrm{mmol} / \mathrm{l}$ Tris-HCl, $\mathrm{pH} 7.4$, $50 \mathrm{mmol} / 1 \mathrm{NaCl}, 25 \mathrm{mmol} / 1 \mathrm{EDTA}, 50 \mathrm{mmol} / \mathrm{l} \mathrm{NaF}, 1.5 \mathrm{mmol} / \mathrm{l}$ $\mathrm{Na}_{3} \mathrm{VO}_{4}, 1 \%$ Triton X-100, 0.1\% SDS, supplemented with protease inhibitors $1 \mathrm{mmol} / 1$ phenylmethylsulfonylfluoride, $10 \mathrm{mg} / \mathrm{l}$ pepstatin, $10 \mathrm{mg} / \mathrm{l}$ aprotinin and $5 \mathrm{mg} / \mathrm{l}$ leupeptin) (all from Sigma). Protein concentrations were determined using the Bradford protein assay. Equal amounts of protein $(50 \mathrm{mg})$ were separated on a $15 \%$ SDS-polyacrylamide gel and transferred to a nitrocellulose membrane (Hybond-C; Amersham, Freiburg, Germany). The membranes were blocked in 5\% non-fat dry milk in TBS for $1 \mathrm{~h}$ at room temperature and probed with anti-HTLV-1 Tax (sc-34096), anti-JNK-1 (sc-1648) or with anti-EGR-1 (sc-110) antibody (dilution 1:500; Santa Cruz Biotechnology) overnight at $4^{\circ} \mathrm{C}$. After 3 washings with TBS containing $0.1 \%$ Tween-20, the membranes were incubated with anti-rabbit IgG-horseradish peroxidase (1:5,000; Santa Cruz Biotechnology), and developed by luminal-mediated chemiluminescence (Appylgen Technologies, Inc., China). To confirm equal protein loading, the membranes were reprobed with a 1:1,000 dilution of an anti-actin antibody (Santa Cruz Biotechnology). Densitometric analyses were performed using Scion Image software (31).

DNA fragmentation. DNA fragmentation was determined using a Cell Death Detection ELISA (Roche). Jurkat and Jurkat-Tax cells were treated with apoptotic compounds for the indicated periods of time. Cells were harvested by centrifugation and lysed in $0.5 \mathrm{ml}$ of the lysis buffer provided with the kit. Two milliliters of the extract was used for the ELISA, which was performed as instructed by the manufacturer. The OD at $405 \mathrm{~nm}$ was measured and the fold induction of apoptosis was calculated using untreated cells as control.

Labeling of apoptotic cells with Annexin V. Jurkat cells $(500,000)$ were treated with RA as indicated. The cells were washed with PBS and stained with Annexin V-FITC (Pharmingen) and PI in binding buffer (10 mM HEPES ( $\mathrm{pH} 7.4,140 \mathrm{mM} \mathrm{NaCl} 2.5 \mathrm{mM} \mathrm{CaCl}_{2}$ ) for $15 \mathrm{~min}$ at room temperature in the dark. Cells were subsequently analyzed by flow cytometry (FACSCalibur) for apoptosis (FITC) and viability (PI).

\section{Results}

We previously reported that application of ATRA signaling causes reduction in Tax activity in Jurkat leukemia cells transfected to express the Tax protein (31). However, the role of EGR-1 in Tax-induced T cell proliferation has not been fully understood. To address this issue, the effects of EGR-1 on the transcriptional activity of Tax and JNK proteins in the presence of ATRA signaling pathways were examined.
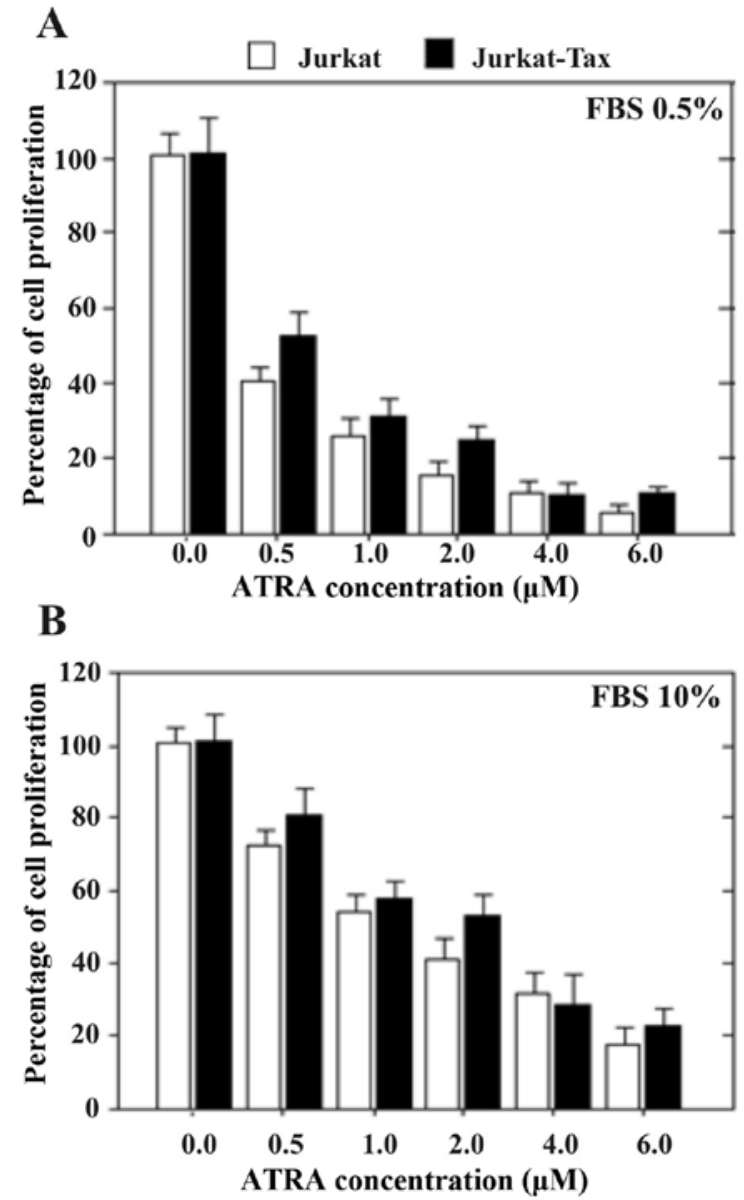

Figure 1. Increasing amounts of serum inhibit the anti-proliferative activity of ATRA. Jurkat cells were treated with increasing concentrations of ATRA, in medium containing $0.5 \%$ FBS (A) or $10 \%$ FBS (B), compared to the proliferation of cell grown in the presence of vehicle (DMSO), used as control $(100 \%)$. After $24 \mathrm{~h}$, cells proliferation was measured by MTT staining. The experiments were repeated four times with triplicates and the mean \pm SD is shown.

Jurkat cells expressing the Tax protein exhibit significantly higher anti-proliferative activity when grown in low concentrations of serum $(0.5 \% \mathrm{FBS})$ in comparison to high serum concentration (10\% FBS). Jurkat cells (wt) and Jurkat cells expressing the Tax protein were grown with increasing concentrations of ATRA for 1, 2, or 3 days in the presence of a low $(0.5 \%)$ or high $(10 \%)$ concentration of serum. Cell growth was measured using the MTT assay. Fig. 1A shows the results obtained in Jurkat and Jurkat-Tax cells after $24 \mathrm{~h}$ of incubation.

Small interfering RNA against EGR-1 did not affect the induction of Tax protein in Jurkat and Jurkat cells transfected to express the Tax protein. EGR-1 is not required for the expression of Tax protein induced by certain stimuli. We investigated whether the siRNA-EGR-1 induced or blocked Tax activity, compared with the induction of EGR-1 and JNK-1 proteins. Cells were incubated for $16 \mathrm{~h}$ in medium containing $0.5 \% \mathrm{FBS}$ prior to ionomycin or TPA stimulation as positive control, or treated with siRNA-control and siRNA against EGR-1 (Fig. 2). A significant activation of Tax (top), EGR-1 (middle) and JNK-1 (bottom) was observed after $120 \mathrm{~min}$ of exposure to the 

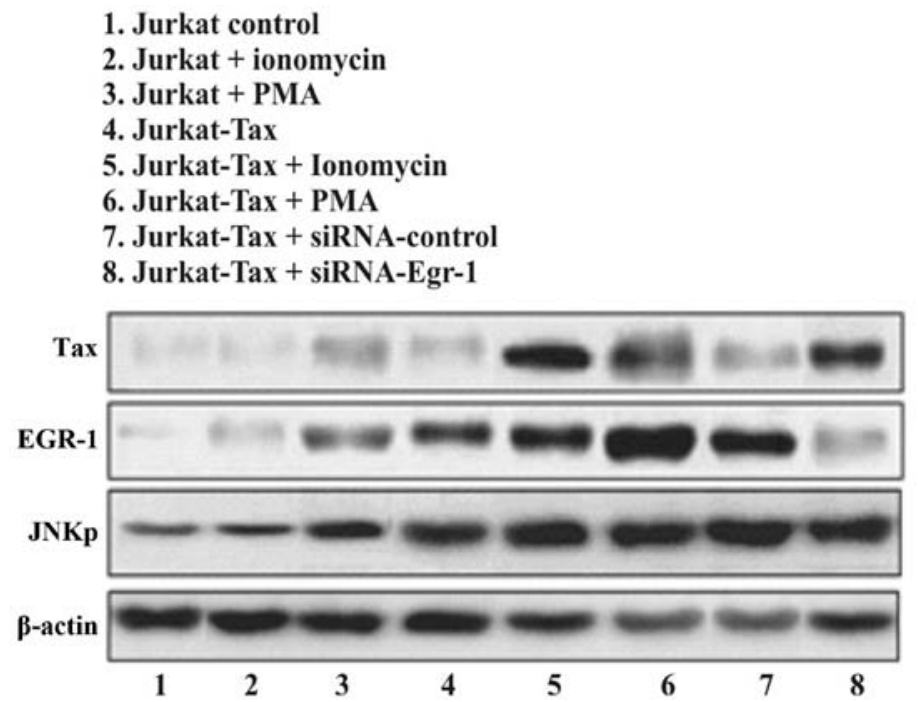

Figure 2. Activation of Tax, EGR-1 and JNK-1 proteins by ionomycin, PMA or siRNA-Egr-1. Jurkat cells were incubated with the given concentration of ionomycin (1 mM), PMA (30 nM) or siRNA-Egr-1 or DMSO as control, for $90 \mathrm{~min}$ in medium containing $0.5 \%$ FBS. Whole cell extracts were prepared and subsequently the levels of Tax, EGR-1 and JNK-1 proteins were examined by western blot analysis. Representative western blot results are presented for Jurkat wild-type and/or Jurkat cells transfected with the Tax expression plasmid. A representative blot of three is shown.
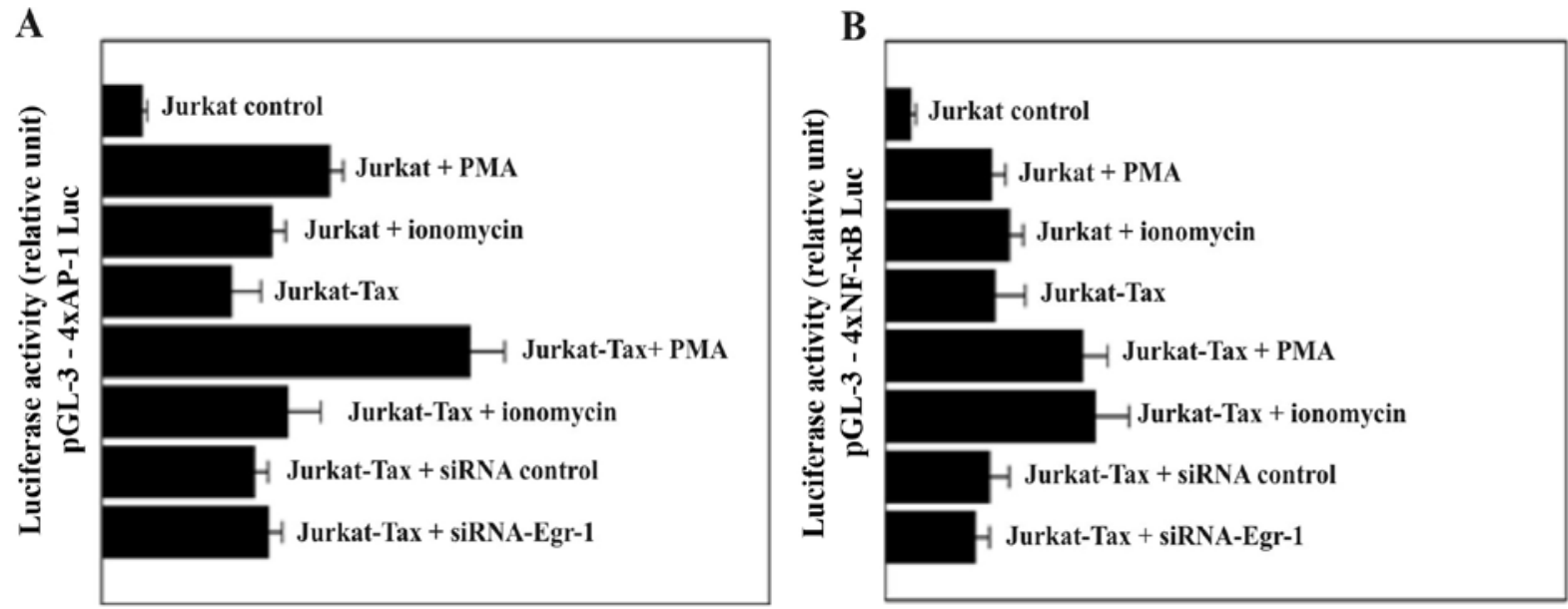

Figure 3. Blocking Egr-1 expression by siRNA did not affect the luciferase activity of the consensus sequences of AP-1 and NF-кB luciferase reporter constructs. Jurkat and Jurkat-Tax cells were transiently transfected with a pGL-3-luciferase plasmid carrying multimers of the consensus sequences of AP-1 (A) (4xCGATTGAGTCAGGGTAACG) or the consensus sequences of NF-kB (B) (4xGGGACTTTCC) and cultured in the presence or absence of PMA (30 nM), ionomycin (1 mM) or siRNA-Egr-1 for $24 \mathrm{~h}$ post-transfection. Luciferase assay is expressed as RLU minus background units alone. All sets of results shown are mean values from three similar experiments. Error bars indicate standard errors of the mean.

stimuli. However, the treatment of cells with siRNA against EGR-1 did not affect the expression of either Tax or JNK-1 proteins (Fig. 2 top and bottom, respectively).

Firefly luciferase activity levels in Jurkat-Tax cells transfected with reporter plasmids containing multiple consensus sequences of $N F-\kappa B$ and $A P-1$ response elements were not affected by inhibition of the EGR-1 expression. We transfected cells with a reporter plasmid containing multiple consensus sequences of 4xAP-1 (Fig. 3A) and/or 4xNF- $\mathrm{kB}$ (Fig. 3B) response elements linked to a minimal promoter controlling transcription of the firefly luciferase cDNA. Both nuclear factors, AP-1 and NF- $\kappa \mathrm{B}$, play important roles in inflammation, immune responses, cell growth and apoptosis. PMA and ionomycin are potent activators of AP-1 and/or NF- $\mathrm{NB}$ responsive genes. In the present study, we demonstrated that transiently transfected cells with the 4xAP-1 (Fig. 3A) exhibited stronger and consistent luciferase expression in response to PMA and ionomycin stimulation compared with cells transfected with the luciferase vector carrying the $4 \times \mathrm{NF} \mathrm{\kappa B}$ (Fig. $3 \mathrm{~B}$ ) response element. At the same time, the expression of luciferase activity in Jurkat-Tax cells was not affected by treatment of the cells with either the siRNA-control or siRNA-Egr-1 (Fig. 3).

Silencing of Egr-1 expression does not block ATRA-induced Jurkat-Tax cell apoptosis but is clearly dependent on the concentration of FBS concentration (0.5 or 10\%). To determine whether the silencing of Egr-1 expression is correlated with the increase or reduction in ATRA-induced Jurkat-Tax cell apoptosis, RNA interference was used. As shown in Fig. 4, 


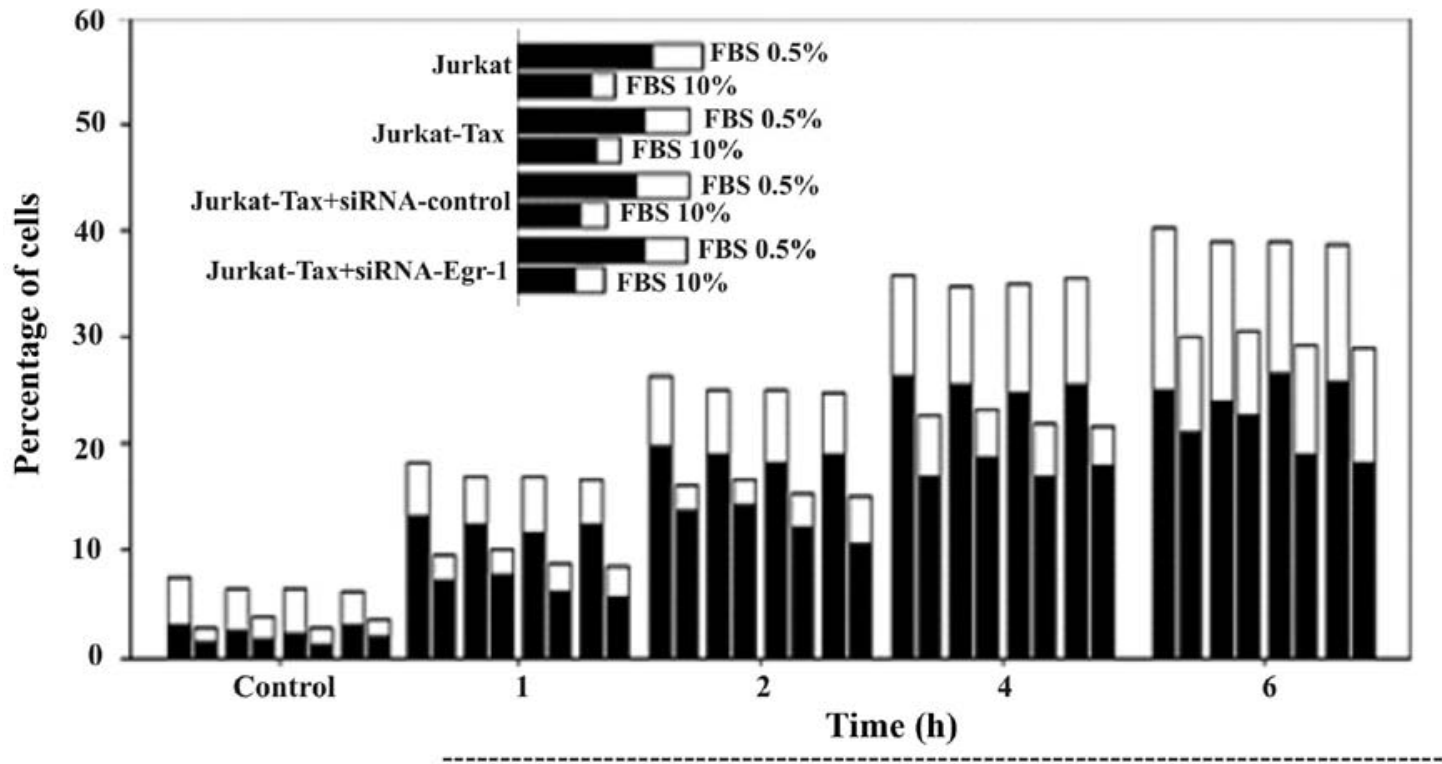

ATRA $8 \mu$ M

Figure 4. Effect of siRNA against Egr-1 treatment on induction of apoptosis by ATRA retinoid in Jurkat and Jurkat-Tax cells. Labeling of apoptotic cells with Annexin V. Cells were incubated with $8 \mu \mathrm{M}$ ATRA in $0.5 \%$ FBS (left columns) or 10\% FBS (right columns) for the indicated periods of time, when apoptosis was determined by double staining with Annexin V-FITC and PI followed by cytometric analysis. The percentages of Annexin V positive/PI negative cells (an indicative of early apoptosis) (black columns) and double positive cells (late apoptosis and/or necrosis) (white columns) are shown. A representative experiment performed in triplicate is shown.

A

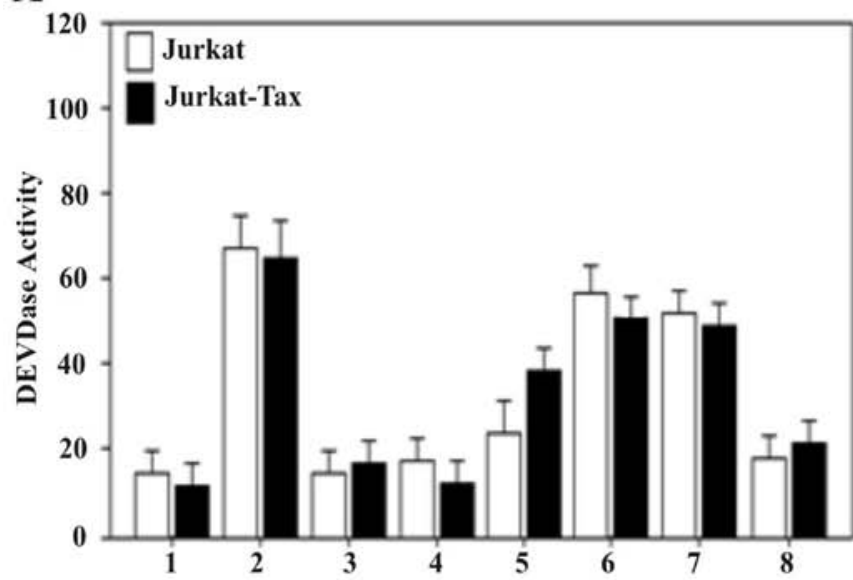

B

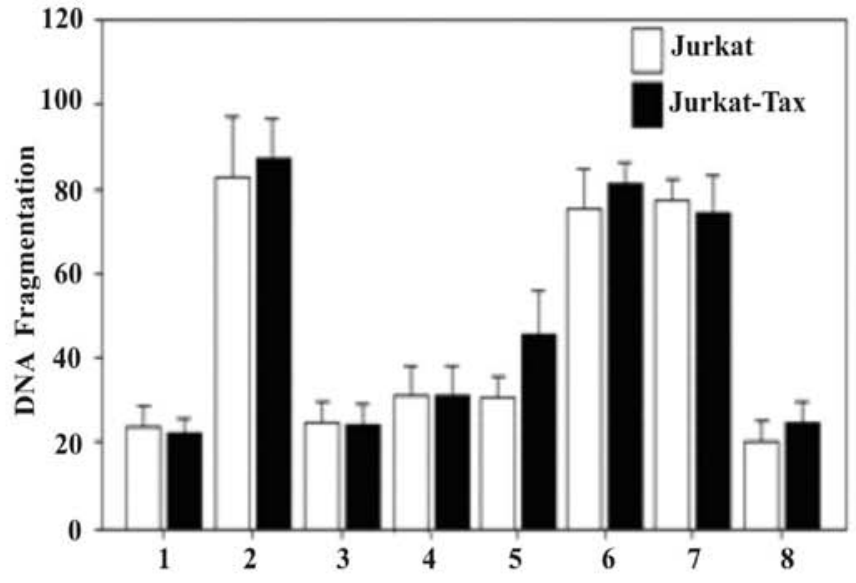

1. Jurkat

2. ATRA

3. SiRNA-control

4. SiRNA-Egr-1

5. SiRNA-JNK-1

6. SiRNA-control+ATRA

7. SiRNA-Egr-1+ATRA

8. SiRNA-JNK-1+ATRA

Figure 5. siRNA against Egr-1 did not prevent ATRA-induced apoptosis in Jurkat and Jurkat-Tax cells. Cells were transfected with either the siRNA-control, siRNA-Egr-1 or siRNA-JNK-1 and incubated at $37^{\circ} \mathrm{C}$ in $5 \% \mathrm{CO}_{2}$ for $48 \mathrm{~h}$ prior to stimulation with ATRA $8 \mu \mathrm{M}$ in medium containing $0.5 \% \mathrm{FBS}$. After $4 \mathrm{~h}$ of ATRA exposure, cells were assayed for DEVDase activity (A) and DNA fragmentation (B). The percentage of DEVDase activity or DNA fragmentation was compared to that measured in the ATRA-treated cells $(100 \%)$. The experiment was repeated three times and represented as mean \pm SD.

transient transfection of a specific siRNA against Egr-1 (siRNA-Egr-1) slightly attenuated ATRA-induced apoptosis in the Jurkat and Jurkat-Tax-cells. However, these results were clearly dependent on the concentration of FBS and on the time of incubation at a given ATRA concentration. As shown in Fig. 4, Jurkat and Jurkat-Tax cells were incubated with either PBS $0.5 \%$ (left columns) or with PBS 10\% (right columns) for the indicated periods of time, and apoptosis was determined by double staining with Annexin V-FITC and PI followed by cytometric analysis. The percentages of 
Annexin V-positive/PI-negative cells (indicative of early apoptosis) (black columns) and double-positive cells (late apoptotic and/or necrotic) (white columns) are shown. Cells incubated with vehicle for $1 \mathrm{~h}$ were used as control. The induction of DEVDase activity was higher when a low concentration of serum was present in the culture medium. The appearance of Annexin V-positive (apoptotic) cells was evident after only $1 \mathrm{~h}$ of incubation with ATRA, and reached a maximum after 4-6 h (Fig. 4). We showed that the percentage of apoptotic cells was higher in the presence of a low serum concentration $(0.5 \%$ FBS) compared with a high serum concentration (10\% FBS).

Induction of apoptosis by ATRA is not altered by inhibition of the EGR-1 pathway. Several studies have demonstrated the inhibitory effect of ATRA on the proliferation of Jurkat cells (31). We examined the ability of ATRA to induce apoptosis in Jurkat and Jurkat-Tax cells by analysis of DEVDase activity (Fig. 5A) and DNA fragmentation (Fig. 5B). To examine whether EGR-1 and/or JNK-1 activities are required for the induction of apoptosis by ATRA in Jurkat-Tax cells, we examined the effect of small interfering RNA inhibitors against Egr-1 and JNK-1 on the induction of apoptosis by ATRA. Jurkat cells were treated with siRNA-Egr-1 or siRNA-JNK-1 or non-silencing siRNA as the control, prior to treatment with $8 \mu \mathrm{M}$ ATRA for $2 \mathrm{~h}$. Fig. 5A shows that the specific siRNA-Egr-1 had no effect on the apoptosis induced by ATRA, as determined by DEVDase activity (Fig. 5A) and by DNA fragmentation (Fig. 5B). In contrast, inhibition of JNK-1 by a specific siRNA reduced ATRA-induced apoptosis (Fig. 5). The results demonstrated that the induction of apoptosis by ATRA in Jurkat-Tax cells was clearly dependent on the presence of JNK kinase activity (Fig. 5).

\section{Discussion}

The HTLV-1 transcriptional activator protein Tax transactivates the expression of many cellular genes in addition to the viral LTR $(1,2,10,11,32)$. Previous studies have demonstrated that the Tax response of the IL-2R $\alpha$ chain promoter is mediated by the activation of NF- $\kappa B(33,34)$. Tax increases the expression of NF- $\kappa \mathrm{B}$ proteins (35) and prolongs its localization to the nucleus where it is transcriptionally active $(35,36)$. Tax also interacts specifically with IкB proteins and induces its degradation $(34,37,38)$, and in vitro studies have shown that several retinoids are strong inducers of apoptosis in a wide variety of cancer cell lines (39-41).

Here, we showed that activation of Tax protein by JNK-1 kinase was not dependent on the presence or not of the early growth response factor-1 gene product. Luciferase assay showed that AP-1 consensus elements were required for strong luciferase activity, while the NF- $\kappa \mathrm{B}$ response element remained low, suggesting that activation of JNK is required for ATRA-induce apoptosis in Jurkat-Tax cells which is necessary for the transcriptional activation of c-jun. There is a strong and sustained activation of JNK-1 and EGR-1 before and after ATRA treatment of Jurkat-Tax cells. JNK activity correlates with the induction of apoptosis, as determined by measurement of DEVDase activity, DNA fragmentation and labelling of cells with Annexin V. Our data demonstrated that the activation or blocking of EGR-1 does not have any effect on the activation and expression of JNK-1 kinase in Jurkat-Tax leukemia cells. Importantly, JNK activation was sufficient for the induction of apoptosis by ATRA, since inhibition of EGR-1 by a specific siRNA had no effect on ATRA-induce apoptosis. As observed, the strong activation of JNK-1 and EGR-1 induced by PMA and ionomycin was further increase in both Jurkat and Jurkat-Tax cells. However, EGR-1 did not appear to be essential for Tax activation, suggesting that it may not be necessary for JNK mediated-Jurkat-Tax cell proliferation. More probably the role of EGR-1 in Tax-induce proliferation of Jurkat cells is independent of JNK-1 kinase activity. Activation of JNK-1 kinase in Jurkat-Tax cells led to the phosphorylation of c-Jun a member of the transcription factor AP-1 (14-18). The results suggest that activation of JNK-1 kinase in Jurkat-Tax cells may lead to the phosphorylation of certain anti-apoptotic proteins promoting cell proliferation. On the other hand, in the presence of ATRA, the JNK kinase activity may lead to the activation of pro-apoptotic protein favoring the induction of apoptosis. The JNK signaling pathway is involved in a variety of cellular responses, and the outcomes of cellular response are varied and complicated. Similar to the JNK pathway, the involvement of EGR-1 in apoptosis is also diverse $(22,23,42,43)$. It has been shown that both EGR-1 and JNK signaling promotes cell death $(21,44)$, whereas it has also been shown that both cascades enhance survival $(42,45)$, cell growth (46), and differentiation (47). These data therefore indicate that the JNK or EGR-1 pathway is required for apoptosis and survival depending on cell types and conditions. Although it is known that JNK kinase participates in the induction of apoptosis and/or cell survival by various chemical drugs, the real contribution of EGR-1 in Tax-transfected Jurkat cells remains to be examined.

\section{Acknowledgements}

We gratefully acknowledge Dr Warner Greene (Gladstone Institute of Virology and Immunology, University of California and San Francisco, CA, USA) for providing the plasmid expressing the wild-type of the Tax protein and the Tax-inducible cell line. The present study was supported by the Biomedical Experimental Laboratory and Intramural Regular Research Grant, UTA-6710-14, University of Tarapacá, AricaChile.

\section{References}

1. Araya N, Sato T, Yagishita N, Ando H, Utsunomiya A, Jacobson S and Yamano Y: Human T-lymphotropic virus type 1 (HTLV-1) and regulatory T cells in HTLV-1-associated neuroinflammatory disease. Viruses 3: 1532-1548, 2011.

2. Hinuma Y, Nagata K, Hanaoka M, Nakai M, Matsumoto T, Kinoshita KI, Shirakawa S and Miyoshi I: Adult T-cell leukemia: Antigen in an ATL cell line and detection of antibodies to the antigen in human sera. Proc Natl Acad Sci USA 78: 6476-6480, 1981.

3. Cann AJ, Rosenblatt JD, Wachsman W, Shah NP and Chen IS: Identification of the gene responsible for human T- cell leukaemia virus transcriptional regulation. Nature 318: 571-574, 1985.

4. Felber BK, Paskalis H, Kleinman-Ewing C, Wong-Staal F and Pavlakis GN: The $\mathrm{pX}$ protein of HTLV-I is a transcriptional activator of its long terminal repeats. Science 229: 675-679, 1985.

5. Sodroski J, Rosen C, Goh WC and Haseltine W: A transcriptional activator protein encoded by the x-lor region of the human T-cell leukemia virus. Science 228: 1430-1434, 1985. 
6. Grassmann R, Berchtold S, Radant I, Alt M, Fleckenstein B, Sodroski JG, Haseltine WA and Ramstedt U: Role of human T-cell leukemia virus type $1 \mathrm{X}$ region proteins in immortalization of primary human lymphocytes in culture. J Virol 66: 4570-4575, 1992.

7. Siekevitz M, Feinberg MB, Holbrook N, Wong-Staal F and Greene WC: Activation of interleukin 2 and interleukin 2 receptor (Tac) promoter expression by the trans activator (tat) gene product of human T-cell leukemia virus, type I. Proc Natl Acad Sci USA 84: 5389-5393, 1987

8. Ballard DW, Bohnlein E, Lowenthal JW, Wano Y, Franza BR and Greene WC: HTLV-I tax induces cellular proteins that activate the kappa B element in the IL-2 receptor alpha gene. Science 241: $1652-1655,1988$

9. Leung KY and Nabel GJ: HTLV-1 transactivator induces interleukin-2 receptor expression through an NF-kappa B-like factor. Nature 333: 776-778, 1988.

10. Franchini G: Molecular mechanisms of human T-cell leukemia/ lymphotropic virus type I infection. Blood 86: 3619-3639, 1995.

11. Franklin AA and Nyborg JK: Mechanisms of Tax regulation of human $\mathrm{T}$ cell leukemia virus type I gene expression. J Biomed Sci 2: 17-29, 1995.

12. Bergers G, Graninger P, Braselmann S, Wrighton C and Busslinger M: Transcriptional activation of the fra-1 gene by AP-1 is mediated by regulatory sequences in the first intron. Mol Cell Biol 15: 3748-3758, 1995.

13. Angel P and Karin M: The role of Jun, Fos and the AP-1 complex in cell-proliferation and transformation. Biochim Biophys Acta 1072: 129-157, 1991

14. Fuchs SY, Xie B, Adler V, Fried VA, Davis RJ and Ronai Z: c-Jun $\mathrm{NH} 2$-terminal kinases target the ubiquitination of their associated transcription factors. J Biol Chem 272: 32163-32168, 1997.

15. Yao M, Nguyen TV and Pike CJ: Beta-amyloid-induced neuronal apoptosis involves c-Jun N-terminal kinase-dependent downregulation of Bcl-w. J Neurosci 25: 1149-1158, 2005.

16. Widmann C, Gibson S, Jarpe MB and Johnson GL: Mitogen-activated protein kinase: Conservation of a three-kinase module from yeast to human. Physiol Rev 79: 143-180, 1999.

17. Xia Z, Dickens M, Raingeaud J, Davis RJ and Greenberg ME: Opposing effects of ERK and JNK-p38 MAP kinases on apoptosis. Science 270: 1326-1331, 1995.

18. Behrens A, Sibilia M and Wagner EF: Amino-terminal phosphorylation of c-Jun regulates stress-induced apoptosis and cellular proliferation. Nat Genet 21: 326-329, 1999.

19. Zhang CC and Shapiro DJ: Activation of the p38 mitogen-activated protein kinase pathway by estrogen or by 4-hydroxytamoxifen is coupled to estrogen receptor-induced apoptosis. J Biol Chem 275 479-486, 2000

20. Yu R, Mandlekar S, Tan TH and Kong AN: Activation of p38 and c-Jun N-terminal kinase pathways and induction of apoptosis by chelerythrine do not require inhibition of protein kinase C. J Biol Chem 275: 9612-9619, 2000.

21. Hoffmann E, Ashouri J, Wolter S, Doerrie A, Dittrich-Breiholz O, Schneider H, Wagner EF, Troppmair J, Mackman N and Kracht M: Transcriptional regulation of EGR-1 by the interleukin-1-JNK-MKK7-c-Jun pathway. J Biol Chem 283: 12120-12128, 2008

22. Parra E, Ferreira J and Ortega A: Overexpression of EGR-1 modulates the activity of NF- $\mathrm{BB}$ and AP-1 in prostate carcinoma PC-3 and LNCaP cell lines. Int J Oncol 39: 345-352, 2011.

23. Parra E and Ferreira J: The effect of siRNA-Egr-1 and camptothecin on growth and chemosensitivity of breast cancer cell lines. Oncol Rep 23: 1159-1165, 2010.

24. Parra E, Ortega A and Saenz L: Down-regulation of Egr-1 by siRNA inhibits growth of human prostate carcinoma cell line PC-3. Oncol Rep 22: 1513-1518, 2009.

25. Liu C, Yao J, Mercola D and Adamson E: The transcription factor EGR-1 directly transactivates the fibronectin gene and enhances attachment of human glioblastoma cell line U251. J Biol Chem 275: 20315-20323, 2000.

26. Calogero A, Arcella A, De Gregorio G, Porcellini A, Mercola D, Liu C, Lombari V, Zani M, Giannini G, Gagliardi FM, et al: The early growth response gene EGR-1 behaves as a suppressor gene that is down-regulated independent of $\mathrm{ARF} / \mathrm{Mdm} 2$ but not p53 alterations in fresh human gliomas. Clin Cancer Res 7 : 2788-2796, 2001.
27. Zhang H, Chen X, Wang J, Guang W, Han W, Zhang H, Tan X and Gu Y: EGR1 decreases the malignancy of human non-small cell lung carcinoma by regulating KRT18 expression. Sci Rep 4: 5416,2014

28. Parra E, Varga M, Hedlund G, Kalland T, Dohlsten M Costimulation by B7-1 and LFA-3 targets distinct nuclear factors that bind to the interleukin-2 promoter: $\mathrm{B} 7-1$ negatively regulates LFA-3-induced NF-AT DNA binding. Mol Cell Biol 17: 1314-1323, 1997.

29. Parra E, Varga M, Sigvardsson M, Hedlund G, Kalland T, Leanderson T, Sjogren $\mathrm{H}$ and Dohlsten M: Costimulation of human $\mathrm{CD}^{+}{ }^{+} \mathrm{T}$ cells with LFA-3 and B7 induce distinct effects on AP-1 and NF-kappa B transcription factors. J Immunol 155 1132-1140, 1995

30. Parra E: Activation of MAP kinase family members triggered by TPA or ionomycin occurs via the protein phosphatase 4 pathway in Jurkat leukemia T cells. Mol Med Rep 5: 773-778, 2012.

31. Parra E and Gutiérrez L: Growth inhibition of Tax-activated human Jurkat leukemia $T$ cells by all-trans retinoic acid requires JNK-1 inhibition. Oncol Rep 29: 387-393, 2013.

32. Azran I, Schavinsky-Khrapunsky Y and Aboud M: Role of Tax protein in human T-cell leukemia virus type-I leukemogenicity. Retrovirology 1: 20,2004

33. Good L, Maggirwar SB and Sun SC: Activation of the IL-2 gene promoter by HTLV-I tax involves induction of NF-AT complexes bound to the CD28-responsive element. EMBO J 15: 3744-3750, 1996.

34. Harhaj EW and Harhaj NS: Mechanisms of persistent NF-kappaB activation by HTLV-I tax. IUBMB Life 57: 83-91, 2005.

35. Li XH, Murphy KM, Palka KT, Surabhi RM and Gaynor RB The human T-cell leukemia virus type-1 Tax protein regulates the activity of the IkappaB kinase complex. J Biol Chem 274: 34417-34424, 1999.

36. Xiao G and Sun SC: Activation of IKKalpha and IKKbeta through their fusion with HTLV-I tax protein. Oncogene 19: 5198-5203, 2000.

37. Petropoulos L, Lin R and Hiscott J: Human T cell leukemia virus type 1 tax protein increases NF-kappa B dimer formation and antagonizes the inhibitory activity of the I kappa B alpha regulatory protein. Virology 225: 52-64, 1996.

38. Verma IM: Nuclear factor (NF)-kappaB proteins: Therapeutic targets. Ann Rheum Dis 63 (Suppl 2): ii57-ii61, 2004.

39. Holmes WF, Soprano DR and Soprano KJ: Synthetic retinoids as inducers of apoptosis in ovarian carcinoma cell lines. J Cell Physiol 199: 317-329, 2004.

40. Zhang XK: Vitamin A and apoptosis in prostate cancer. Endocr Relat Cancer 9: 87-102, 2002.

41. Zhang D, Holmes WF, Wu S, Soprano DR and Soprano KJ: Retinoids and ovarian cancer. J Cell Physiol 185: 1-20, 2000.

42. Liu C, Rangnekar VM, Adamson E and Mercola D: Suppression of growth and transformation and induction of apoptosis by EGR-1. Cancer Gene Ther 5: 3-28, 1998.

43. Virolle T, Adamson ED, Baron V, Birle D, Mercola D, Mustelin T and de Belle I: The Egr-1 transcription factor directly activates PTEN during irradiation-induced signalling. Nat Cell Biol 3: 1124-1128, 2001

44. Chen L, Wang S, Zhou Y, Wu X, Entin I, Epstein J, Yaccoby S, Xiong W, Barlogie B, Shaughnessy JD Jr, et al: Identification of early growth response protein 1 (EGR-1) as a novel target for JUN-induced apoptosis in multiple myeloma. Blood 115: 61-70, 2010.

45. Park JM, Greten FR, Li ZW and Karin M: Macrophage apoptosis by anthrax lethal factor through p38 MAP kinase inhibition. Science 297: 2048-2051, 2002.

46. Juretic N, Santibanez JF, Hurtado C and Martinez J: ERK 1,2 and p38 pathways are involved in the proliferative stimuli mediated by urokinase in osteoblastic SaOS-2 cell line. J Cell Biochem 83: 92-98, 2001.

47. Halawani D, Mondeh R, Stanton LA and Beier F: p38 MAP kinase signaling is necessary for rat chondrosarcoma cell proliferation. Oncogene 23: 3726-3731, 2004. 